\title{
ON PONTRYAGIN DUALITY
}

\author{
by B. J. DAY
}

(Received 9 June, 1977)

Introduction. The main aim of this article is to discuss the relationship between Pontryagin duality and pro-objects. The basic idea arises from K. H. Hofmann's articles [7] and [8] where it is shown that the elementary abelian (Lie) groups are "dense" in the category of locally compact hausdorff abelian groups.

We commence with a good symmetric monoidal closed category $\mathscr{V}$ and a full sub-V $\mathscr{V}$-category $\mathscr{A} \subset \mathscr{V}$ of "elementary" objects. We then build pro- $\mathscr{A}$-objects as suitable projective limits of these elementary objects. This is done with a view to extending Pontryagin duality to pro- $\mathscr{A}$-objects once it holds in $\mathscr{A}$ with respect to some basic dualising object which we call $\Omega$. The actual pro- $\not A$-objects constructed are relative to a subcategory $\mathscr{E}$ of $\mathscr{V}_{0}$ which, in practice, is usually taken to be some good class of epimorphisms in $\mathscr{V}_{0}$. This is done in Sections 2 and 3.

In Section 4 we discuss to what extent projective limits of pro- $\not A$-objects are again pro- $\mathscr{A}$-objects. This at least explains one of Kaplan's results [10]; namely, that the product of locally compact hausdorff abelian groups satisfies Pontryagin duality. Kaplan's second result [11] remains to be fitted into this context.

In the examples of Section 5 we apply the results of the preceding sections to prove that Pontryagin duality holds for any abelian group object in the category of compactly generated spaces which is a suitable projective limit of its elementary Lie quotients. We also reproduce the duality of Hofman, Mislove and Stralka [9] between semilattices and compact zero-dimensional semilattices.

For basic notation and terminology we refer the reader to Day and Kelly [3], Eilenberg and Kelly [5] and Mac Lane [12].

1. Preliminaries. Let $\mathscr{V}=\left(\mathscr{V}_{0}, V, \otimes, I,[-,-], \ldots\right)$ be a complete and cocomplete symmetric monodial closed category in the sense of Eilenberg and Kelly [5]. This means that we have at our disposal the calculus of $\mathscr{V}$-ends discussed in Day and Kelly [3] and in Dubuc [4].

Let $\mathscr{E}$ s denote "the" category of small sets and set maps. We denote the $X$-fold power, respectively copower, of $X \in \mathscr{E}_{n}$ s with $C \in \mathscr{V}$ by $\{X, C\}$, respectively $X$. $C$.

We now assume that $V: \mathscr{V}_{0} \rightarrow \mathscr{E}_{n s}$ is faithful. The effect of this assumption is the following.

Lemma 1.1. Suppose $\mathscr{A}$ is a small $\mathscr{V}$-category and $S: \mathscr{A}^{\mathrm{op}} \otimes \mathscr{A} \rightarrow \mathscr{V}$ is a $\mathscr{V}$-functor. Let $S^{\prime}$ denote the composite.

Then

$$
V_{*} \mathscr{A}^{\mathrm{OP}} \times V_{*} \mathscr{A} \stackrel{\hat{\mathrm{V}}_{*}}{\longrightarrow} V_{*}\left(\mathscr{A}^{\mathrm{op}} \otimes \mathscr{A}\right) \stackrel{V_{*} \mathrm{~S}}{\longrightarrow} V_{*} \mathscr{V}=\mathscr{V}_{0} .
$$

$$
\int_{A \in V_{*} \infty \mathcal{A}} S^{\prime}(A A) \cong \int_{A \in \mathscr{A}} S(A A)
$$

Glasgow Math. J. 20 (1979) 15-24. 
Proof. Following the notation of Eilenberg and Kelly [5] we write $\mathscr{A}_{0}=V_{*} \mathscr{A}$. Because $V: \mathscr{V}_{0} \rightarrow \mathscr{E}_{n s}$ is faithful a family $\alpha_{A}: C \rightarrow S(A A)$ is $\mathscr{V}$-natural in $A \in \mathscr{A}$ if and only if it is Ens-natural in $A \in \mathscr{A}_{0}$ since

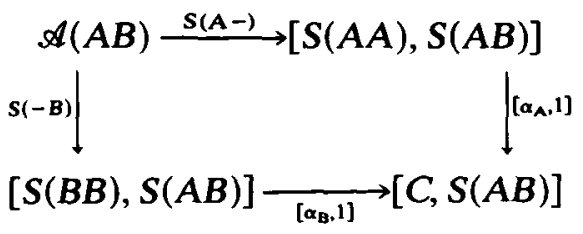

commutes if and only if

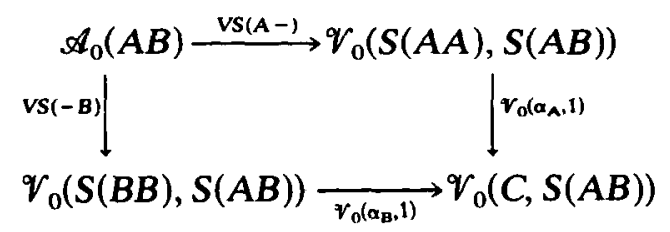

commutes. Thus the equaliser of the canonical pair

$$
\prod_{A \in \mathscr{A}} S(A A) \Longrightarrow \prod_{A, B \in \mathscr{A}}[\mathscr{A}(A, B), S(A B)]
$$

which is, by definition, $\int_{A \in \mathscr{A}} S(A A)$ coincides with the equaliser of the canonical pair

$$
\prod_{A \in \mathscr{A}_{0}} S(A A)=\prod_{A, B \in \mathscr{A}_{0}}\left\{\mathscr{A}_{0}(A, B), S(A B)\right\}
$$

which is, by definition, $\int_{A \in A_{0}} S^{\prime}(A A)$.

Henceforth we shall denote $S^{\prime}(A B)$ simply by $S(A B)$.

The assumption that $V: \mathscr{V}_{0} \rightarrow \mathscr{E}$ ns is faithful allows us, in effect, to "mix" $\mathscr{V}$-ends with ordinary $\mathscr{E}$ ss-ends.

2. Pro- $\mathscr{A}$-objects. Let $\mathscr{A} \subset \mathcal{V}$ be a full sub- $\mathscr{V}$-category of $\mathscr{V}$. Let $\mathscr{E}$ be a subcategory of $\mathscr{V}_{0}$ and let $\mathscr{H}=\mathscr{E} \cap \mathscr{A}_{0}$.

DefinITION 2.1. (i) A pro- $\mathscr{A}$-object in $\mathscr{V}$ relative to $\mathscr{E}$ is an object $C \in \mathscr{V}$ such that $C \cong \int_{A \in \mathscr{K}}\{\mathscr{E}(C, A), A\}$.

(ii) A strong pro- $\mathscr{A}$-object in $\mathscr{V}$ relative to $\mathscr{E}$ is a pro- $\mathscr{A}$-object $C \in \mathscr{V}$ such that $\int^{A \in \mathscr{X}} \mathscr{E}(C, A) .[A, B] \rightarrow[C, B]$ is an epimorphism for all $B \in \mathscr{A}$.

The $\mathscr{V}$-category of pro- $\mathscr{A}$-objects is denoted by $\mathscr{P} \mathscr{A}(\mathscr{C})$ while the $\mathscr{V}$-category of strong pro- $\mathscr{A}$-objects is denoted by $\mathscr{P P} \mathscr{A}(\mathscr{E})$.

LEMMA 2.2. $\mathscr{A} \subset \mathscr{P P} \mathscr{A}(\mathscr{E})$.

Proof. If $A^{\prime} \in \mathscr{A}$ then $A^{\prime} \cong \int_{A \in \mathscr{C}}\left\{\mathscr{H}\left(A^{\prime}, A\right), A\right\}$ by the representation theorem applied to $A \in \mathscr{H}, \quad \cong \int_{A \in \mathscr{H}}\left\{\mathscr{C}\left(A^{\prime}, A\right), A\right\}$. Similarly $\int^{A \in \mathscr{K}} \mathscr{E}\left(A^{\prime}, A\right) .[A, B]=$ $\int^{A \in \mathscr{K}} \mathscr{H}\left(A^{\prime}, A\right) .[A, B] \cong\left[A^{\prime}, B\right]$ by the representation theorem applied to $A \in \mathscr{H}$.

THEOREM 2.3. The inclusion $\mathscr{A} \subset \mathscr{S P P} \mathscr{A}(\mathscr{E})$ is $\mathscr{V}$-codense (=V-coadequate). 
Proof. For $\mathscr{V}$-codensity we require that $C \cong \int_{A \in \mathscr{A}}[[C, A], A]$ for all $C \in \mathscr{S P P A}(\mathscr{E})$. But, by Definition 2.1(ii), there is a monomorphism

$$
[[C, B], B] \rightarrow\left[\int^{A \in \mathscr{H}} \mathscr{E}(C, A) .[A, B], B\right]
$$

for each $B \in \mathscr{A}$. This gives us a monomorphism

$$
\int_{B \in \mathscr{A}_{0}}[[C, B], B] \rightarrow \int_{B \in \mathscr{A}_{0}}\left[\int^{A \in \mathscr{H}} \mathscr{E}(C, A) \cdot[A, B], B\right]
$$

since limits of monomorphisms are monomorphisms. Moreover, the codomain of this monomorphism becomes:

$$
\begin{aligned}
\int_{B \in \mathcal{A}_{0}}\left[\int^{A \in \mathscr{K}} \mathscr{E}(C, A) .[A, B], B\right] & \cong \int_{B \in \mathscr{A}_{0}} \int_{A \in \mathscr{H}}\{\mathscr{C}(C, A),[[A, B], B]\} \\
\cong & \int_{A \in \mathscr{H}} \int_{B \in \mathscr{A}_{0}}\{\mathscr{C}(C, A),[[A, B], B]\} \\
& \text { on interchanging ends, } \\
\cong & \left.\int_{A \in \mathscr{X}}\left\{\mathscr{C}(C, A), \int_{B \in \mathscr{A}_{0}}[[A, B], B]\right]\right\}
\end{aligned}
$$

where $\int_{B \in \mathscr{S}_{0}}[[A, B], B] \cong \int_{B \in \mathscr{A}}[[A, B], B]$ (by Lemma 1.1$) \cong A$ by the $\mathscr{V}$-representation theorem applied to $B \in \mathscr{A}$. Thus we obtain a monomorphism

$$
m: \int_{B \in \mathscr{A}_{0}}[[C, B], B] \rightarrow \int_{A \in \mathscr{K}}\{\mathscr{E}(C, A), A\} \cong C
$$

by Definition 2.1(i). However, this monomorphism is left inverse to the canonical transformation $\eta$ from $C$ to $\int_{B \in A_{0}}[[C, B], B]$ by consideration of the following diagram:

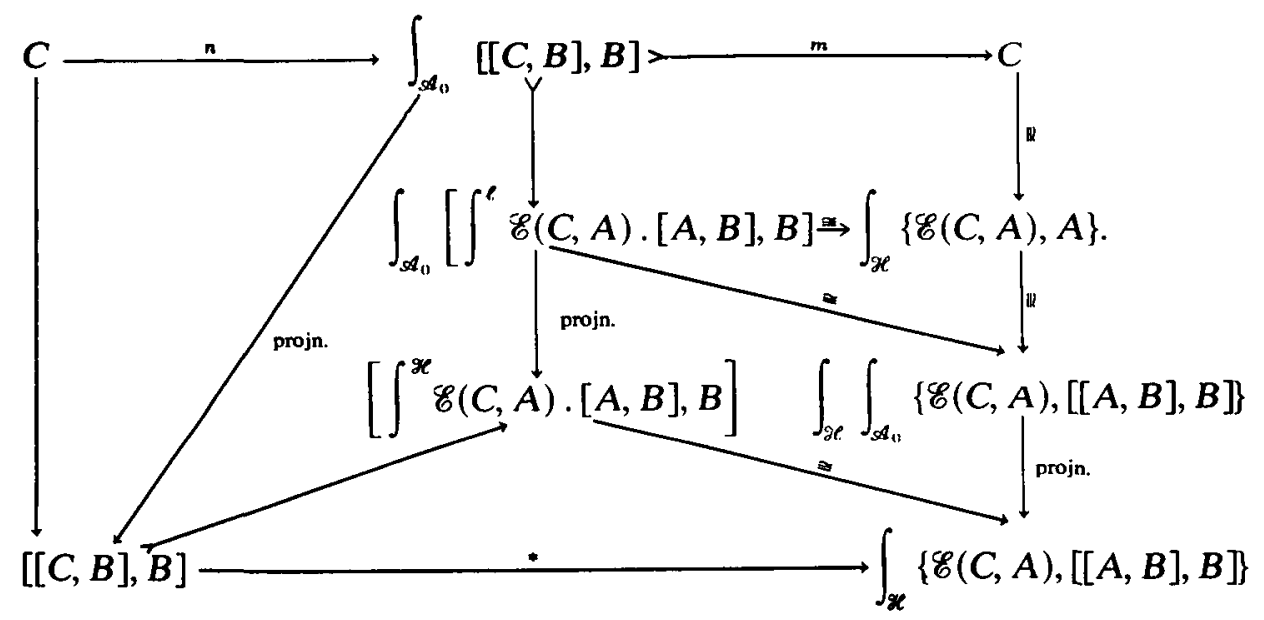


where $*$ commutes for obvious reasons (project both legs at $A \in \mathscr{H}$ and $f \in \mathscr{E}(C, A)$ ). Thus $C \cong \int_{B \in \mathcal{A}_{0}}[[C, B], B] \cong \int_{B \in \mathscr{A}}[[C, B], B]$ by Lemma 1.1 , as required.

3. Duality. Given $\mathscr{A} \subset \mathcal{V}$ we can form the Pontryagin closure of $\mathscr{A}$ :

$$
\dot{\mathscr{A}}=\left\{C \in \mathscr{V} ; C \cong \int_{A \in \mathscr{A}}[[C, A], A] \text { in } \mathscr{V}\right\} \text {. }
$$

Proposition 3.1. $\mathscr{A} \subset \bar{A}$ and $\bar{A}=\bar{A}$.

Proof. If $B \in \mathscr{A}$ then $B \cong \int_{A \in \mathscr{A}}[[B, A], A]$ by the $\mathscr{V}$-representation theorem applied to $A \in \mathscr{A}$. Thus $\mathscr{A} \subset \overline{\mathscr{A}}$ so $\bar{A} \subset \bar{A}$. But $C \in \bar{A}$ implies $C \cong \int_{A^{\prime} \in \mathscr{A}}\left[\left[C, A^{\prime}\right], A^{\prime}\right]$ where $A^{\prime} \in \bar{A}$ implies $A^{\prime} \cong \int_{A \in \mathscr{A}}\left[\left[A^{\prime}, A\right], A\right]$. So

$$
\begin{aligned}
C & \cong \int_{A^{\prime} \in \overline{\mathscr{A}}}\left[\left[C, A^{\prime}\right], \int_{A_{A \in \mathscr{A}}}\left[\left[A^{\prime}, A\right], A\right]\right] \\
& \cong \int_{A \in \mathscr{A}}\left[\int^{A^{\prime} \in \bar{A}}\left[C, A^{\prime}\right] \otimes\left[A^{\prime}, A\right], A\right] \\
& \cong \int_{A \in \mathscr{A}}[[C, A], A]
\end{aligned}
$$

by the $\mathscr{V}$-representation theorem applied to $A^{\prime} \in \overline{\mathscr{A}}$.

Corollary 3.2. The inclusion $A \subset \bar{A}$ is $\mathscr{V}$-codense and if $\mathscr{C} \subset \mathscr{V}$ with $\mathscr{A} \subset \mathscr{C}$ being $\mathcal{V}$-codense then $\mathscr{C} \subset \overline{\mathcal{A}}$.

Given $\mathscr{A} \subset \mathcal{V}$ and $\Omega \in \mathscr{A}$ such that $[[A, \Omega], \Omega] \cong A$ we have:

Proposition 3.3. Pontryagin duality with respect to $\Omega \in \mathscr{A}$ holds in $\bar{A}$.

Proof. If $C \in \bar{A}$ then

$$
\begin{aligned}
C & \cong \int_{A \in \mathscr{A}}[[C, A], A] \\
& \cong \int_{A \in \mathscr{A}}[[C, A],[[A, \Omega], \Omega]] \\
& \cong\left[\int^{A \in \mathscr{A}}[C, A] \otimes[A, \Omega], \Omega\right] \\
& \cong[[C, \Omega], \Omega]
\end{aligned}
$$

by the $\mathscr{V}$-representation theorem applied to $A \in \mathscr{A}$.

Corollary 3.4. Pontryagin duality with respect to $\Omega$ holds in $\mathscr{S P A}(\mathscr{E})$.

Proof. By Theorem 2.3.

We now examine the case where $\int^{A \in \mathscr{K}} \mathscr{C}(C, A) .[A, B] \rightarrow[C, B]$ is an isomorphism for 
all $C \in \mathscr{V}$ and $B \in \mathscr{A}$. When this is so $\mathscr{P} \mathscr{A}(\mathscr{E})$ and $\mathscr{S} \mathscr{P} \mathscr{A}(\mathscr{E})$ coincide and we can define an endofunctor $P: \mathscr{V} \rightarrow \mathscr{V}$ by any one of the formulas

$$
P C=\int_{A \in \mathscr{X}}\{\mathscr{E}(C, A), A\} \cong \int_{A \in \mathscr{A}}[[C, A], A] \cong[[C, \Omega], \Omega] .
$$

We recall from Day [1] that a category $\mathscr{C}$ is $\mathcal{M}$-complete if $\mathcal{M}$ is a subcategory of monomorphisms in $\mathscr{C}$ such that $\mathscr{C}$ has the following inverse limits and $\mathcal{M}$ contains each monomorphism so formed:

(a) equalisers of pairs of morphisms,

(b) pullbacks of $\mu$-monomorphisms,

(c) the intersection of any family of $\mathcal{M}$-monomorphisms with a common codomain. A functor $T: \mathscr{C} \rightarrow \mathscr{B}$ is $\mathscr{M}$-continuous if it preserves these inverse limits in $\mathscr{C}$.

Proposition 3.5. If $\mathscr{V}$ is $\mu$-complete for some system $\mu$ of monomorphisms then $\mathscr{P} \mathscr{A}(\mathscr{E}) \subset \mathcal{V}$ is reflective if $[-, \Omega]: \mathscr{V}^{\mathrm{op}} \rightarrow \mathscr{V}$ preserves suitable colimits.

Proof. Basically we require that $[-, \Omega]^{\text {op }}: \mathscr{V} \rightarrow \mathscr{V}^{\text {op }}$ be $\mathcal{M}$-continuous and that $[-, \Omega]: \mathscr{V}^{\mathrm{op}} \rightarrow \mathscr{V}$ preserve linear colimits. The effect of the first requirement is that $\mathscr{P} \mathscr{A}(\mathscr{E})$ is $\mu$-complete and the inclusion $\mathscr{P A}(\mathscr{E}) \subset \mathscr{V}$ is $M$-continuous. Thus we can apply Day [1, Theorem 2.2] provided $P: \mathscr{V} \rightarrow \mathscr{V}$ is a suitable boundary functor. But the canonical morphism $\eta_{C}: C \rightarrow P C$ gives us:

$$
C \stackrel{n_{c}}{\longrightarrow} P C \stackrel{n_{P C}}{\longrightarrow} P^{2} C \longrightarrow \ldots \longrightarrow P^{n} C \longrightarrow \ldots
$$

where $P^{n+1} C=\left[\left[P^{n} C, \Omega\right], \Omega\right]$. If $[-, \Omega]$ preserves linear colimits then $P^{\omega} C=$ colim $P^{n+1} C=\operatorname{colim}\left[\left[P^{n} C, \Omega\right], \Omega\right]=\left[\lim \left[P^{n} C, \Omega\right], \Omega\right]=\left[\left[\operatorname{colim} P^{n} C, \Omega\right], \Omega\right]=\left[\left[P^{\omega} C, \Omega\right], \Omega\right]$. Thus $P^{\omega} C$ lies in $\mathscr{P A}(\mathscr{C})$ for all $C \in \mathcal{V}$. This implies (by Day [1, Theorem 2.2]) that $\mathscr{P A}(\mathscr{E}) \subset \mathcal{V}$ is reflective and the reflection of $C \in \mathcal{V}$ is the intersection in $\mathscr{V}$ of all the $\mathscr{P} \mathscr{A}(\mathscr{C})$ - $M$-subobjects of $P^{\omega} C$ through which the resultant canonical transformation $\beta_{C}: C \rightarrow P^{\omega} C$ factors.

We recall that $\Omega \in \mathscr{V}$ is said to be a strong $\mathscr{V}$-cogenerator for $\mathscr{V}$ if $[-, \Omega]: \mathscr{V}^{\mathrm{op}} \rightarrow \mathscr{V}$ reflects isomorphisms.

COROLlaRY 3.6. If $\Omega$ is a strong $\mathscr{V}$-cogenerator for $\mathscr{V}$ then $\mathscr{P} \mathscr{A}(\mathscr{E})=\mathscr{V}$ if and only if $[-, \Omega]: \mathscr{V}^{\mathrm{OP}} \rightarrow \mathscr{V}$ preserves colimits.

Proof. If $[-, \Omega]$ preserves colimits then $\mathscr{P} \mathscr{A}(\mathscr{E}) \subset \mathscr{V}$ is $\mathscr{V}$-reflective hence is closed under $\mathscr{V}$-limits. Thus, if $\Omega$ is a strong $\mathscr{V}$-cogenerator then every object of $\mathscr{V}$ is a $\mathscr{V}$-limit of copies of $\Omega$. Thus $\mathscr{P A}(\mathscr{C})=\mathscr{V}$. Conversely, if $\mathscr{P A}(\mathscr{C})=\mathscr{V}$ then Pontryagin duality holds in $\mathcal{V}$ with respect to $\Omega$. Thus $\operatorname{colim}\left[A_{\lambda}, \Omega\right] \cong\left[\lim A_{\lambda}, \Omega\right]$ because $\left[\operatorname{colim}\left[A_{\lambda}, \Omega\right], \Omega\right] \cong$ $\lim \left[\left[A_{\lambda}, \Omega\right], \Omega\right] \cong \lim A_{\lambda} \cong\left[\left[\lim A_{\lambda}, \Omega\right], \Omega\right]$ where $\Omega$ is a strong $\mathscr{V}$-cogenerator so that $[-, \Omega]$ reflects isomorphisms. 
4. Strong $\mathscr{A}$-limits. A strong $\mathscr{A}$-limit relative to $\mathscr{E}$ is a $\operatorname{limit} \lim C_{\lambda}$ in $\mathscr{V}_{0}$ such that the canonical morphisms

$$
\sum \mathscr{E}\left(C_{\lambda}, A\right) \rightarrow \mathscr{E}\left(\lim C_{\lambda}, A\right), \quad \sum\left[C_{\lambda}, A\right] \rightarrow\left[\lim C_{\lambda}, A\right]
$$

are epimorphisms for all $A \in \mathscr{A}$.

Proposition 4.1. A strong $\mathscr{A}$-limit of strong pro-A-objects is a strong pro-A-object.

Proof. The morphism $\sum \mathscr{E}\left(C_{\lambda}, A\right) \rightarrow \mathscr{E}\left(\lim C_{\lambda}, A\right)$ is a surjection if and only if the canonical morphism colim $\mathscr{E}\left(C_{\lambda}, A\right) \rightarrow \mathscr{E}\left(\lim C_{\lambda}, A\right)$ is an epimorphism. This gives a monomorphism

$$
\begin{aligned}
\int_{A \in \mathscr{K}}\left\{\mathscr{E}\left(\lim C_{\lambda}, A\right), A\right\} \rightarrow & \int_{A \in \mathscr{H}}\left\{\operatorname{colim} \mathscr{E}\left(C_{\lambda}, A\right), A\right\} \\
& \cong \lim \int_{A \in \mathscr{H}}\left\{\mathscr{E}\left(C_{\lambda}, A\right), A\right\} \\
& \cong \lim C_{\lambda} .
\end{aligned}
$$

Moreover, this monomorphism is left inverse to the canonical morphism from $\lim C_{\lambda}$ to $\int_{A \in \mathscr{C}}\left\{\mathscr{E}\left(\lim C_{\lambda}, A\right), A\right\}$ hence it is an isomorphism. The fact that $\lim C_{\lambda}$ is a strong pro- $\mathscr{A}$-object now follows from consideration of the following diagram:

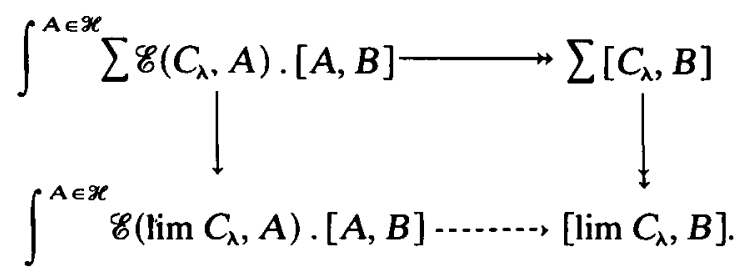

Here the dotted arrow is an epimorphism because the diagonal is an epimorphism.

\section{Examples.}

EXAMPLE 5.1. Let $\mathscr{V}$ be the symmetric monoidal closed category $\mathscr{C} \& \mathscr{\&}$ of abelian group objects in the category $\mathscr{C}$ of all convergence spaces (i.e. limit spaces). Let $\mathscr{A}=\left\{\mathbf{R}^{m} \oplus(\mathbf{R} / \mathbf{Z})^{n} \oplus G ; m, n \in \mathbf{N}\right.$ and $G$ discrete $\}$. Let $\Omega=\mathbf{R} / \mathbf{Z}$ and let $\mathscr{C}$ be the category of identification maps.

Proposition 5.1.1. Each locally compact hausdorff abelian group is a strong pro- $\mathscr{A}$ object.

Proof. Each locally compact hausdorff abelian group $C$ is a pro- $\mathscr{A}$-object by the Lie-group approximation theorem: see Hofmann [7]. Secondly, each continuous 
homomorphism $f: C \rightarrow B, B \in \mathscr{A}$, factors as

$$
C \stackrel{e}{\longrightarrow} C / \operatorname{ker} f \stackrel{m}{\longrightarrow} B
$$

where $C / \operatorname{ker} f$ is a locally compact hausdorff group, hence is a Lie group (see Hochschild [6, Chapter VIII]), so $C / \operatorname{ker} f \in \mathscr{A}$. This implies that $\int^{A \in \mathscr{K}} \mathscr{E}(C, A) .[A, B] \rightarrow[C, B]$ is a surjection, as required.

Corollary 5.1.2. Pontryagin duality in $\mathscr{C} A \mathbb{B}$ holds for locally compact hausdorff abelian groups.

A strong projective limit in $\mathscr{T}$, the category of topological spaces and continuous maps, is a limit $\lim _{\lambda \in \Lambda} C_{\lambda}$ over a cofiltered index category $\Lambda$ such that each projection $p_{\lambda}: \lim C_{\lambda} \rightarrow$ $C_{\lambda}$ is an identification map. For example, a product $\Pi C_{\lambda}$ may be regarded as a strong limit cofiltered by the set of finite subsets of $\Lambda$.

Lemma 5.1.3. Given a strong projective limit in $\mathscr{T A} \&$, with projections $p_{\lambda}: \lim C_{\lambda} \rightarrow C_{\lambda}$, the collection $\left\{\operatorname{ker} p_{\lambda} ; \lambda \in \Lambda\right\}$ is a filter base on $\lim C_{\lambda}$ which converges to zero.

Proof. Since $\Lambda$ is cofiltered the collection $\left\{p_{\lambda}^{-1}(V) ; V\right.$ open in $\left.C_{\lambda}\right\}$ is a base for the topology on $\lim C_{\mathrm{\lambda}}$ in $\mathscr{T} \mathscr{A} \&$. Thus $\left\{\operatorname{ker} p_{\lambda}\right\} \rightarrow 0$.

Proposition 5.1.4. A strong projective limit $\lim C_{\lambda}$ in $\mathscr{T} \mathscr{A} b$ is a strong $\mathscr{A}$-limit in $\mathscr{C} \mathscr{A} \mathbb{b}$.

Proof. For each $A \in \mathscr{A}$, the canonical morphisms $\sum \mathscr{E}\left(C_{\lambda}, A\right) \rightarrow \mathscr{E}\left(\lim C_{\lambda}, A\right)$ and $\sum\left[C_{\lambda}, A\right] \rightarrow\left[\lim C_{\lambda}, A\right]$ are epimorphisms by Lemma 5.1.3 and the fact that each $A \in \mathscr{A}$ has no small subgroups.

Corollary 5.1.5. A product of locally compact hausdorff groups satisfies Pontryagin duality in $\mathscr{C} \mathbb{A} \mathbb{B}$.

EXAMPLE 5.2. Let $\mathscr{V}=\mathscr{K} \mathscr{A} b_{2}$ be the category of hausdorff abelian group objects in the category $\mathscr{K}$ of $k$-spaces. With $\mathscr{A}$ and $\Omega$ as in Example 5.1 let $\mathscr{E}$ consist of all epimorphisms in $\mathscr{K} \mathscr{A} \mathfrak{b}_{2}$.

Propostrion 5.2.1. Pontryagin duality holds for pro- $\mathscr{A}$-objects

Proof. Each pro- $\mathscr{A}$-object is now strong because any morphism $f: C \rightarrow B$ factors as

$$
C \stackrel{e}{\longrightarrow} \overline{C / \operatorname{ker} f} \stackrel{m}{\longrightarrow} B
$$

where $e$ is an epimorphism and $m$ is a closed subspace. Thus $\overline{C / \operatorname{ker} f} \in \mathscr{A}$.

It is actually possible to show that each locally compact hausdorff abelian group is a pro- $\mathscr{A}$-object for this $\mathscr{E}$ on $\mathscr{K} \mathscr{A} b_{2}$; this we leave to the reader.

Example 5.3. Let $K$ be a discrete field and let $\mathscr{V}$ be the category of $K$-vector spaces in $\mathscr{K}$. Let $\mathscr{A} \subset \mathcal{V}$ be the full subcategory determined by $\left\{K^{n} ; n \in \mathbf{N}\right\}$. Then Pontryagin 
duality holds in $\mathscr{A}$ with respect to $\Omega=K$. Let $\mathscr{E}$ be the category of strong epimorphisms in $\mathscr{V}$. Then each map $f: C \rightarrow K^{n}$ factors as

$$
C \stackrel{e}{\longrightarrow} \mathrm{C} / \mathrm{ker} f \stackrel{m}{\longrightarrow} K^{n}
$$

where $e$ is a strong epimorphism and $C /$ ker $f$ is of the form $K^{p}$ for some $p \in \mathbf{N}$. Thus $\int^{A \in \mathscr{H}} \mathscr{E}(C, A) .[A, B] \rightarrow[C, B]$ is an epimorphism for all $C \in \mathscr{V}$ and $B \in \mathscr{A}$.

Proposition 5.3.1. Pontryagin duality with respect to $\Omega=K$ holds for pro- $A$-objects.

EXAMPLE 5.4. Let $K$ be a topological field in $\mathscr{K}$ and let $\mathscr{V}$ be the category of $K$-vector spaces in $\mathscr{K}$. Let $\mathscr{A}$ consist of 0 and $K$ and let $\Omega=K$. Let $\mathscr{E}$ be the category of epimorphisms in $\mathscr{V}$. Now each map $f: C \rightarrow B, B \in \mathscr{A}$, factors

$$
C \stackrel{e}{\longrightarrow} C / \operatorname{ker} f \stackrel{m}{\longrightarrow} B
$$

where $e$ is an epimorphism and $C /$ ker $f$ is either 0 or $K$.

Proposition 5.4.1. Pontryagin duality with respect to $\Omega=K$ holds for pro- $\mathscr{A}$-objects.

EXAMPLE 5.5. Let $\mathscr{V}$ be the symmetric monoidal closed category of semilattices in $\mathscr{K}$ (see Hofmann, Mislove and Stralka [9]). Let $\mathscr{A}$ be the finite discrete semilattices in $\mathscr{V}$ and let $\mathscr{E}$ be the category of strong epimorphisms. Also let $\Omega=2 \in \mathscr{A}$. factors as

Once again every pro- $\mathscr{A}$-object is strong because any morphism $f: C \rightarrow B$ in $\mathscr{V}$

$$
C \stackrel{e}{\longrightarrow} C / \operatorname{ker} f \stackrel{m}{\longrightarrow} B
$$

where $C / \operatorname{ker} f$ is finite since $B$ is finite.

For any compact zero-dimensional semilattice $C$ in $\mathscr{K}$ we have

$$
C \cong \int_{A \in \mathscr{X}}\{\mathscr{E}(C, A), A\}
$$

because this is true in the category of topological semilattices (see Numakura [13] and Hofmann, Mislove and Stralka [9]). Thus, if $\mathscr{Z}$ denotes the category of compact zerodimensional semilattices we have:

Proposition 5.5.1. Pontryagin duality with respect to $\Omega=2$ holds for each object of $\mathscr{Z}$.

Proof. Pontryagin duality with respect to $\Omega=2$ holds in $\mathscr{A}$ by [9, Chapter 1 , Lemma 3.8].

In his example $\mathscr{Z}$ has an explicit dual category, namely the category $\mathscr{S}$ of semilattices and semilattice morphisms (see [9, Chapter I]). This is so because we have $(\varepsilon, \eta): F \leftrightarrow$ $R \cdot \mathscr{Z}^{\text {op }} \rightarrow \mathscr{S}$ given by $R=[-, \Omega]$ and $F=[-, \Omega]^{\text {op }}$. Because Pontryagin duality holds in $\mathscr{Z}$ 
we have $\varepsilon: F R \cong 1: \mathscr{Z} \rightarrow \mathscr{X}$. To prove $\eta: 1 \rightarrow R F: \mathscr{S} \rightarrow \mathscr{S}$ is an isomorphism note that $F: \mathscr{S} \rightarrow \mathscr{Z}^{\text {op }}$ reflects isomorphisms because $\Omega=2$ is a (strong) cogenerator in $\mathscr{S}$ (see [9, Chapter I, Proposition 1.4]). Thus it suffices to prove that $F \eta: F \rightarrow F R F$ is an isomorphism. But this follows from the triangle identity

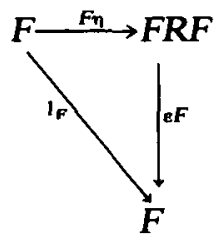

In [9, Chapter I] it is shown that $\mathscr{S}$ is symmetric monoidal closed. This puts us in the situation of Proposition 3.5 and Corollary 3.6 because $\int^{A \in \mathscr{K}} \mathscr{E}(C, A) \times \mathscr{Y}(A, B) \rightarrow \mathscr{S}(C, B)$ is easily seen to be an isomorphism for all $B \in \mathscr{A}$ (as before) and $C \in \mathscr{S} ; \mathscr{E}$ denotes the category of (strong) epimorphisms. Noting again that 2 is a strong cogenerator of $\mathscr{S}$ we have that $\mathscr{P} \mathscr{A}(\mathscr{E})=\mathscr{S}$. Thus Pontryagin duality for $\mathscr{S}$ could be shown directly by proving that $[-, 2]: \mathscr{S}^{\circ \mathrm{p}} \rightarrow \mathscr{S}$ preserves colimits; as it is, this is a consequence of duality in $\mathscr{S}$ as derived from the duality in $\mathscr{Z}$.

ExAmpLE 5.6. Let $V=R-\mu o d$ be the category of $R$-modules over a principal ideal domain $R$. Let $\mathscr{A}$ be determined by the free $R$-modules of finite rank, and let $\Omega=R$. Then Pontryagin duality holds in $\mathscr{A}$ with respect to $\Omega=R$. If $\mathscr{E}$ is the category of (strong) epimorphisms in $\mathscr{V}=R-\mu_{o d}$ then $\int^{A \in \mathscr{X}} \mathscr{E}(C, A) .[A, B] \rightarrow[C, B]$ is an epimorphism for all $C \in R-M_{\text {ad }}$ and $B \in \mathscr{A}$. A pro- $\mathscr{A}$-object (= a strong pro-SA-object) is called a pro-free $R$-module.

Proposrrion 5.6.1. Pontryagin duality with respect to $\Omega=R$ holds for pro-free $R$ modules.

Example 5.7. It is clear that the calculations in Sections 1, 2, 3 and 4 can be carried out with $\mathscr{E} n s$ replaced by an arbitrary base category $W$ which is symmetric monoidal closed and complete and cocomplete. As an example, let $W$ be $\mathscr{A} \&$, the category of abelian groups. Now let $R$ be a commutative topological ring in $\mathscr{K}$. Let $\mathscr{V}$ be $R$-modules in $\mathscr{K}$ and let $\mathscr{A}$ comprise $R$ alone as a full subcategory of $\mathscr{V}$. Let $\mathscr{E}$ be all "maps" in $\mathscr{V}_{0}$. (now a $\mathscr{W}$-category). Then every pro- $\mathscr{A}$-object is strong and $R^{n}$ is a pro- $\mathscr{A}$-object for all $n \in \mathbf{N}$ because

$$
R^{n} \cong \int_{A \in \mathscr{K}}\left\{\mathscr{V}_{0}\left(R^{n}, A\right), A\right\}
$$

since $\mathscr{V}_{0}\left(R^{n}, A\right) \cong \oplus \mathscr{V}_{0}(R, A)$. Here of course $\{X, A\}$ denotes $\mathscr{A} b$-cotensoring of $X \in \mathscr{A} \&$ with $\mathscr{A} \in \mathscr{V}$.

EXAMPLE 5.8. It is worth noting to what extent Example 5.5 can be generalised. Let $\mathscr{V}$ be the category of algebras in $\mathscr{K}$ for some commutative algebraic $\mathscr{K}$-theory and let $\mathscr{A}$ be the category of finite discrete algebras in $\mathscr{V}$. Then, by Theorem $2.3, \mathscr{A}$ is $\mathscr{V}$-codense in 
the full sub- $\mathscr{V}$-category $\mathscr{P} \mathscr{A}(\mathscr{E})$ of pro-finite algebras in $\mathscr{V}$ ( $\mathscr{E}$ is the category of strong epimorphisms and all pro- $\mathscr{A}$-objects relative to this $\mathscr{E}$ are strong). Thus $C \cong \int_{n}[[C, n], n]$ for $C$ pro-finite. This may be regarded as a form of Pontryagin duality in which there is generally no basic dualising object $\Omega$ in $\mathscr{A}$. The actual duality is between $\mathscr{P} \mathscr{A}(\mathscr{E})$ and a full sub- $\mathscr{V}$-category of the $\mathscr{V}$-functor category $[\mathscr{A}, \mathscr{V}]$.

Examples are easily obtained. For instance let $\mathcal{V}$ be the category of algebras for the theory of commutative semigroups or the theory of distributive lattices. Then, by Numakura [13], this form of Pontryagin duality holds for the compact zero-dimensional objects of $\mathscr{V}$. For further examples see Hofmann [8].

\section{REFERENCES}

1. Brian Day, On adjoint-functor factorisation, in Category Seminar Sydney 1972/73, Lecture Notes in Mathematics 420 (Springer-Verlag, 1974), 1-19.

2. B. J. Day, Density presentations of functors, Bull. Austral. Math. Soc. 16 (1977), 427-448.

3. B. J. Day and G. M. Kelly, Enriched functor categories, in Reports of the Midwest Category Seminar III, Lecture Notes in Mathematics 106 (Springer-Verlag, 1969), 178-191.

4. Eduardo J. Dubuc, Kan extensions in enriched category theory, Lecture Notes in Mathematics 145 (Springer-Verlag, 1970).

5. Samuel Eilenberg and G. Max Kelly, Closed categories, in Proceedings of the Conference on Categorical Algebra, La Jolla, California, 1965 (Springer-Verlag, 1966), 421-562.

6. G. Hochschild, The structure of Lie groups, (Holden-Day Inc., 1965).

7. Karl Heinrich Hofmann, Categories with convergence, exponential functors, and cohomology of compact abelian groups, Math. Z. 104 (1968), 106-140.

8. Karl Heinrich Hofmann, Category theoretical methods in topological algebra, in Categorical topology Mannheim 1975 (Springer-Verlag, 1976), 345-403.

9. Karl Heinrich Hofmann, Michael Mislove and Albert Stralka, The Pontryagin duality of compact O-dimensional semilattices and its applications, Lecture Notes in Mathematics 396 (Springer-Verlag, 1974).

10. Samuel Kaplan, Extensions of the Pontryagin duality I: infinite products, Duke Math. J. 15 (1948), 649-658.

11. Samuel Kaplan, Extensions of the Pontryagin duality II: direct and inverse sequences, Duke Math. J. 17 (1950), 419-435.

12. S. Mac Lane, Categories for the working mathematician, Graduate Texts in Mathematics 5 (Springer-Verlag, 1971).

13. K. Numakura, Theorems on compact totally disconnected semigroups and lattices, Proc. Amer. Math. Soc. 8 (1957), 623-626.

Department of Pure Mathematics

UNIVERSITY OF SYDNEY

N.S.W. 2006

Australia 